\title{
Pattern and Risk Assessment of Bedsore in Hospitalized Patients
}

\author{
Md Ruhul Amin' \\ Md Monoarul Haque ${ }^{2^{*}}$ \\ Pradip Kumar Saha ${ }^{3}$ \\ Sumaiya Zabin Eusuf Zai ${ }^{4}$ \\ Sarder Mahmud Hossain ${ }^{4}$ \\ Sanjida Akter ${ }^{5}$
}

'Department of Physiotherapy

SAIC

Dhaka, Bangladesh.

${ }^{2}$ Department of Community Nutrition Faculty of Public Health Bangladesh University of Health Sciences (BUHS) Dhaka, Bangladesh.

${ }^{3}$ Department of Physiotherapy National Institute of Traumatology and Orthopedic Rehabilitation (NITOR) Dhaka, Bangladesh.

${ }^{4}$ Department of Public Health Northern University

Dhaka, Bangladesh.

${ }^{5}$ Department of Forensic Medicine Delta Medical College Dhaka, Bangladesh.

${ }^{*}$ Correspondence to:

\section{Md Monoarul Haque}

Fellow

Department of Community Nutrition, Faculty of Public Health Bangladesh University of Health Sciences (BUHS)

125/1, Darus Salam, Mirpur, Dhaka-1216, Bangladesh Cell: +8801915839550

e-mail: monoarmunna@yahoo.com

\begin{abstract}
Objective: This descriptive type of cross sectional study was conducted to assess pattern and risk factors of bedsore in hospital admitted patients in Dhaka city with a sample size of 114. Methods: A pretested, modified, questionnaire was used to collect the data. All the data were entered and analyzed by using Statistical Package of Social Science 16.0 versions. Results: Study found that 17.5\%, 41.2\%, 27.2\% and $14 \%$ of the respondents belonged to age group of 1-20 years, 21-40 years, 41-60 years and 61-80 years respectively with mean age $37.97+16.909$ years. Responsible diseases for bedsore were spinal cord injury $(41.2 \%)$, fracture $(30 \%)$, stroke $(24 \%)$, unconsciousness (7.2\%) and GBS (2.8\%) respectively. Study revealed that common area of bedsore were back of the sacrum (57\%), back of the scapula $(34.2 \%)$, medial aspect of knee joint $(22.8 \%)$, malleoli $(21.1 \%)$, greater trochanter of femur $(15.8 \%)$, external occipital protuberance (14\%), olecranon process of ulna $(9.6 \%)$, above the coccyx $(9.6 \%)$, iliac crest $(7 \%)$, spine of the scapula (3.5\%), posterior superior iliac joint $(2.6 \%)$ and sacro-iliac joint $(1.8 \%)$ respectively. Study also found that superficial and deep type of bedsore were $58.8 \%$ and $41.2 \%$ consecutively. Besides study revealed that $26.3 \%$ of the respondents were diabetic, $31.6 \%$ obese, $28.1 \%$ suffering from malnutrition, $61.4 \%$ did not use pneumatic bed and $7.2 \%$ patients did not maintain proper positioning 2 hourly and these were the risk factors of this study. Significant association was found between type of bed sore and use of pneumatic bed $(\mathrm{P}=0.000<0.05)$ as well as nutritional status $(\mathrm{P}=0.004<0.05)$. Conclusions: Changing position and use of pneumatic bed was best methods of prevention of bedsore.
\end{abstract}

Key words: Pneumatic bed; risk factors; spinal cord injury; stroke.

\section{BACKGROUND}

Bedsores are a significant health burden, but little is known of the impact on the quality of life of the sufferer. They mainly affect older people, Pressure ulcers produce endless pain; pressure ulcers produce a restricted life; coping with a pressure ulcer. Several patients also reported that pain was exacerbated by their pressure relieving equipment and at dressing change. Patients found that the pressure ulcer restricted their activities and had an impact on their families. In addition, for some, the restrictions delayed their rehabilitation ${ }^{1}$. Pressure ulcers are a common and painful health condition, particularly among people who are elderly or physically impaired. Despite our knowledge of how to prevent pressure ulcers, and improvements in treatment, pressure ulcers remain prevalent and impose a significant burden on financial and labor resources in the healthcare industry. Under nutrition is a risk factor, and nutrition therapy plays a crucial role in pressure ulcer treatment. Micronutrients should be replaced if depleted, but routine supplementation of vitamins and minerals in all pressure ulcer patients is not warranted ${ }^{2}$. Cumulative incidence of Pressure ulcer more in acute hospital admitted patients than bedside in home sitting. The relative rates in rehabilitation and nursing home settings were 1.4 (95\% CI 0.8-2.3) and 1.3 (95\% CI 0.8-2.1), respectively ${ }^{3}$. 
To estimate the frequency of use of pressure-redistributing support surfaces among hip fracture patients and to determine whether higher pressure ulcer risk is associated with greater pressure-redistributing support surfaces use. Patients $(\mathrm{n}=658)$ aged 65 years who had surgery for hip fracture were examined by research nurses at baseline and on alternating days for 21 days ${ }^{4}$. Study was found that the mean age of the study was 44.51. Regarding to sex it was found that the highest percentages of the study samples were male $60.4 \%$. It presented that nurses performance were used bed sheets and observe signs and symptoms of sores sites for most of patients as pressure ulcer prevention constituted $100 \%$ and $62.5 \%{ }^{5}$. Although no gold standard for preventing or treating pressure ulcers has been established, data from clinical trials indicate specific efforts are worthwhile. Preventive strategies include recognizing risk, decreasing the effects of pressure, assessing nutritional status, avoiding excessive bed rest, and preserving the integrity of the skin. Treatment principles include assessing the severity of the wound; reducing pressure, friction, and shear forces; optimizing wound care; removing necrotic debris; managing bacterial contamination; and correcting nutritional deficits $^{6}$. Intervention strategies included PU-specific changes in combination with educational strategies. Most studies reported patient outcome measures, while fewer reported nursing process of care measures. For nearly all the studies, the authors concluded that the intervention had a positive effect ${ }^{7}$. Critically ill patients are at high risk for pressure ulcer development due to their high acuity and the invasive nature of the multiple interventions and therapies they receive. With reported incidence rates of bedsore development in the adult critical care population as high as $56 \%$; the identification of patients at high risk of bedsore development is essential. This paper will explore the association between bedsore development and risk factors ${ }^{8}$. Pressure ulcer risk assessment is a component of the assessment process used to identify individuals at risk of developing a pressure ulcer. Use of a risk assessment tool is recommended by many international pressure ulcer prevention guidelines however it is not known whether using a risk assessment tool makes a difference to patient outcomes. A review was conducted to clarify the role of pressure ulcer risk assessment in clinical practice?. This generally well-conducted review concluded that commonly used instruments can predict which patients are more likely to develop a pressure ulcer. There were no clear differences in test accuracy between methods. Advanced static support surfaces were more effective than standard mattresses for reducing risk of pressure ulcers. The review conclusions seem appropriate ${ }^{10}$. Most of the pressure ulcers were stage 2, and the majority was in the sacral area or on the heels. In multivariable analysis, pressure ulcer incidence was significantly associated with incontinence, difficulty turning in bed, nursing home residence prior to admission, recent hospitalization, and poor nutritional status $^{11}$. To identify resident, wound, and treatment characteristics associated with pressure ulcer healing in longterm care residents. In this sample of nursing facility residents, increasing age, male gender, dry skin, urinary and fecal

use of moist dressings and adequate nutritional support are strong predictors of pressure ulcer healing ${ }^{12}$. The overall prevalence of pressure ulcers was $27 \%$. Multivariate analysis showed a statistically significant positive association between high-risk condition of pressure ulcer and previous stroke, previous trauma, cardiovascular diseases. The risk of pressure ulcers according to number of full-time nurses and auxiliary staff per 10 beds lower than five was marginally statistically significant ${ }^{13}$. There is no decrease in pressure ulcer incidence was found which might be attributed to use of an assessment scale. However, the use of scales increases the intensity and effectiveness of prevention interventions. The Braden Scale shows optimal validation and the best sensitivity/specificity balance $^{14}$. Absence of pressure ulcers is increasingly being used as an indicator of quality nursing care, based on the premise that pressure ulcers are preventable. Pressure ulcer incidents were recorded in groups of patients who underwent surgery with use of a standard foam bed mattress; use of a fluid, pressure-reducing bed mattress; after a comprehensive educational program on pressure ulcer prevention was presented $^{15}$.

\section{MATERIAL \& METHODS}

Descriptive type cross-sectional study was conducted to determine the pattern and risk factors of bedsore with 114 samples. The study site was Dhaka Medical College Hospital, National Institute of Traumatology and Orthopedic Rehabilitation situated in Shamolly and Metropolitan Medical Centre, Mohakhali, of Dhaka city. The study period was conducted for six months started from June 2013 to December 2013. Non randomized purposive sampling method was applied for data collection. A pre tested modified semistructured questionnaire which were prepared on the basis of objectives and different variables of this study. The collected data was edited by checking rechecking analyzed by using the software SPSS 16.0 version (Chicago). Then analyzed data were presented according to the variables of the study.

\section{RESULTS}

Analysis of socio-demographic variables and table 1 showed that $17.5 \%, 41.2 \%, 27.2 \%$ and $14 \%$ of the respondents belonged to age group of 1-20years, 21-40 years, 41-60 years and $61-80$ years respectively with mean age $37.97+16.909$ years.

Table 1: Distribution of the respondents by age $(n=114)$

\begin{tabular}{lcc} 
Age & Frequency & Percent \\
$1-20$ & 20 & 17.5 \\
$21-40$ & 47 & 41.2 \\
$41-60$ & 31 & 27.2 \\
$61-80$ & 16 & 14.0 \\
Total & 114 & 100.0 \\
\hline
\end{tabular}


Table 2 showed that most of the respondents $77.2 \%$ are Male and rest of them $22.8 \%$ are Female of them of the respondents are primary $39.5 \%$, followed by Secondary $20.2 \%$, Higher Secondary $12.3 \%$, Graduate $18.4 \%$ and Post Graduate and above $9.6 \%$ respectively ,Among the respondents Married $72.8 \%$, unmarried $21.9 \%$, Widow/ Widowed $3.5 \%$ and only are Divorced/ Separate $1.8 \%$ respectively. It is reveals that occupation of the respondents is student $21.9 \%$, service holder $22.8 \%$, employer $15.8 \%$, worker $31.6 \%$ and rest of them are retread person $7.9 \%$ respectively.

Table 2: Distribution of the respondents by sex $(n=114)$

\begin{tabular}{lcc} 
Sex & Frequency & Percent \\
Male & 88 & 77.2 \\
Female & 26 & 22.8 \\
Total & 114 & 100.0 \\
\hline
\end{tabular}

Table 3 revealed that common area of bedsore of the respondents were followed by back of the sacrum $57 \%$, back of the scapula $34.2 \%$, medial aspect of knee joint $22.8 \%$, malleoli $21.1 \%$, greater trochanter of femur $15.8 \%$, external occipital protuberance $14 \%$, above the coccyx and olecranon process of ulna $9.6 \%$, iliac crest $7 \%$, spine of the scapula $3.5 \%$, posterior superior iliac joint $2.6 \%$, sacro-iliac joint $1.8 \%$, and respectively.

Table 3: Distribution of the respondents by common area of bedsore (Multiple Responses)

\begin{tabular}{lcc} 
Variables & Frequency & Percent \\
External occipital protuberance & 16 & 14.0 \\
Spine of the scapula & 4 & 3.5 \\
Back of the scapula & 39 & 34.2 \\
Olecranon process of ulna & 11 & 9.6 \\
Iliac crest & 8 & 7.0 \\
Posterior superior iliac joint & 3 & 2.6 \\
Sacro-iliac joint & 2 & 1.8 \\
Back of the sacrum & 65 & 57.0 \\
Above the coccyx & 11 & 9.6 \\
Greater trochanter of femur & 18 & 15.8 \\
Medial aspect of knee joint & 26 & 22.8 \\
Malleoli & 24 & 21.1 \\
\hline
\end{tabular}

Figure 1 found that superficial and deep type of bedsore were $58.8 \%$ and $41.2 \%$ respectively.

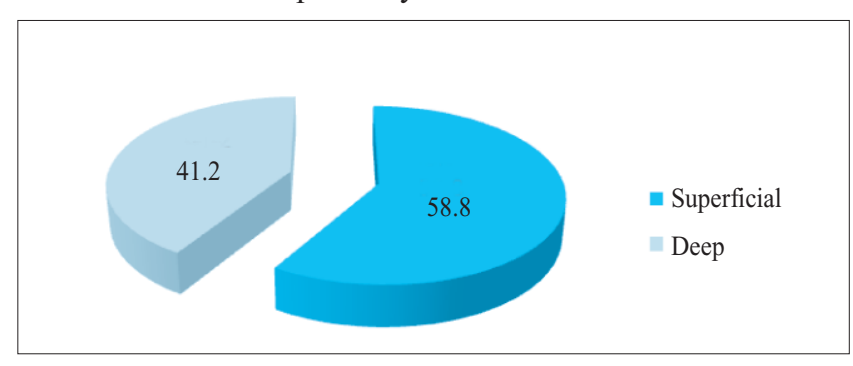

Figure 1: Distribution of the respondents by type of bed sore $(n=114)$
Table 4 revealed that several risk factors influenced the develop and severity of bedsore that were $61.4 \%$ did not use pneumatic bed (air mattress), 31.6\% obese, 28.1\% malnuorished, 26.3\% the respondents were diabetic and $7.2 \%$ patient did not maintain proper positioning 2 hourly.

Table 4: Distribution of the respondents by risk factors $(n=114)$

$\begin{array}{lcc}\text { Risk factors } & \text { Frequency } & \text { Percent } \\ \text { Diabetic } & 30 & 26.3 \\ \text { Obese } & 36 & 31.6 \\ \text { Malnutrition } & 32 & 28.1 \\ \text { Do not use pneumatic bed } & 70 & 61.4 \\ \text { Lack of positioning 2 hourly } & 9 & 7.2\end{array}$

Figure 2 reveled that responsible disease for bedsore were spinal cord injury $41.2 \%$, fracture $30 \%$, stroke $24 \%$, unconsciousness $7.2 \%$, GBS $2.8 \%$.

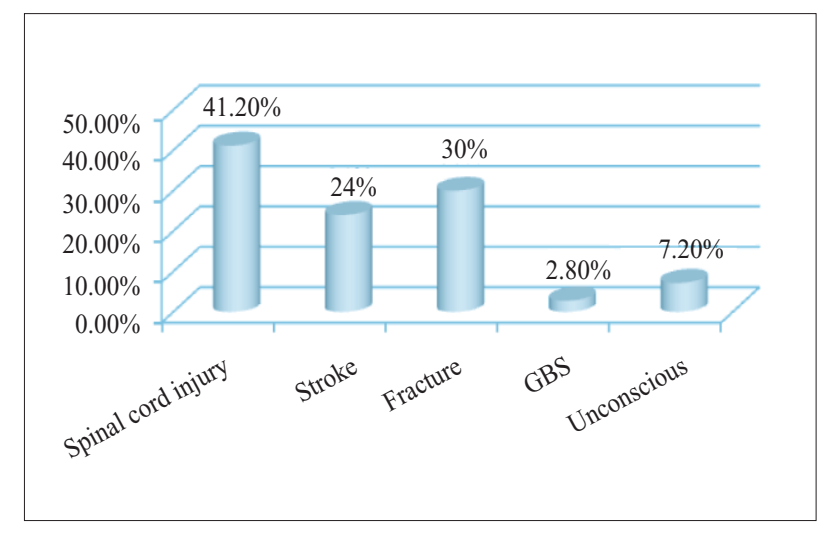

Figure 2: Distribution of the respondents by responsible disease for bedsore (Multiple Responses)

Table 5 revealed that a highly significant association was found between types of bedsore and use pneumatic bed $(\mathrm{P}=0.000<0.05)$.

Table 5: Distribution of respondents by association between type of bedsore and use pneumatic bed $(n=114)$

\begin{tabular}{|c|c|c|c|c|}
\hline \multirow{2}{*}{ Types of bed sore } & \multicolumn{2}{|c|}{ Use Pneumatic bed } & \multirow[t]{2}{*}{ Total } & \multirow[t]{2}{*}{ P-Value } \\
\hline & Yes & No & & \\
\hline Superficial & 12 & 55 & 67 & 0.000 \\
\hline Deep & 32 & 15 & 47 & \\
\hline Total & 44 & 70 & 114 & \\
\hline
\end{tabular}

Table 6 revealed that a significant association was found between type of bedsore and nutritional status of patients $(\mathrm{P}=0.004<0.05)$. 
Table 6: Distribution of respondents by association between type of bedsore and nutritional status of patients $(n=114)$

\begin{tabular}{lcccc} 
Type of bed sore & $\begin{array}{c}\text { Nutritional status of patients } \\
\text { Yes }\end{array}$ & Notal & P-Value \\
Superficial & 55 & 12 & 67 & 0.004 \\
Deep & 27 & 20 & 47 & \\
Total & 82 & 32 & 114 & \\
\hline
\end{tabular}

\section{DISCUSSION}

Study revealed that common area of bedsore were back of the sacrum $57 \%$, back of the scapula $34.2 \%$, medial aspect of knee joint $22.8 \%$, malleoli $21.1 \%$, greater trochanter of femur $15.8 \%$ this findings was similar to the study carried out by the Mona Baumgarten, David Margolis, et al editors ${ }^{4}$. Responsible diseases for bedsore were spinal cord injury $41.2 \%$, fracture $30 \%$, stroke $24 \%$, unconscious $7.2 \%$ and GBS $2.8 \%$ respectively which was also similar to the study of A Capon, $\mathrm{N}$ Pavoni, et al editors ${ }^{13}$. The present study found that $58.8 \%$ superficial type of bedsore and $41.2 \%$ was deep.
Study revealed that $26.3 \%$ of the respondents were diabetic, $31.6 \%$ obese, $28.1 \%$ suffered from malnutrition, $61.4 \%$ did not use pneumatic bed and $7.2 \%$ patients did not maintain proper positioning 2 hourly this findings was supported by the study of Sewchuk D, Padula C, Osborne $E^{15}$. There were statistically significant association between type of bad sore with nutritional status of patient and use pneumatic bed $(\mathrm{P}=0.004<0.05$ and $0.000<0.05)$ and this findings was similar to the study carried out by the M Baumgarten, DJ Margolis, Localio, et al editors ${ }^{11}$.

\section{CONCLUSION}

The present study found pattern and risk factor bedsore, such as obesity, diabetic, malnutrition, did not use pneumatic bed and not maintain proper positioning, bony prominent area of the body in different position especially supine. Above all we concluded that minimizing risk factors and proper physiotherapy care may reduce incidence of bedsore in hospital admitted patients.

\section{DISCLOSURE}

All the authors declared no competing interest. 


\section{REFERENCES}

1. Hopkins A, Dealey C, Bale S, Defloor T, Worboys F. Patient stories of living with a pressure ulcer. J Adv Nurs. 2006 ;56(4):345-353.

2. Jennifer Doley, Jennifer Doley. Nutrition Management of Pressure Ulcers. Nutr Clin Pract February 2010;25(1): 50-60.

3. Mona Baumgarten, David J. Margolis, Denise L. Orwig, Michelle D. Shardell,et al editors. Pressure ulcers in elderly hip fracture patients across the continuum of care. J Am Geriatr Soc. 2009; 57(5): 863-870.

4. Mona Baumgarten, David Margolis, Denise Orwig, William Hawkes, et al editors. Use of pressure-redistributing support surfaces among elderly hip fracture patients across the continuum of care: adherence to pressure ulcer prevention guidelines. Gerontologist. 2010; 50(2): 253-262.

5. Inshrah Roshdy Mohamed and Esraa Esam-Eldin Mohamed. Nursing Practices for patients at risk to the pressure sores in Minia University Hospital. Journal of American Science 2013;9(4).

6. Thomas DR. Prevention and treatment of pressure ulcers: what works? what doesn't? Cleve Clin J Med. 2001;68(8):7047, 710-14, 717-722.

7. Soban LM, Hempel S, Munjas BA, Miles J and Rubenstein LV. Preventing pressure ulcers in hospitals: A systematic review of nurse-focused quality improvement interventions. Jt Comm J Qual Patient Saf. 2011;37(6):245-252.

8. Nahla Tayyib, Fiona Coye and Peter Lewis. Pressure ulcers in the adult intensive care unit: a literature review of patient risk factors and risk assessment scales. Journal of Nursing Education and Practice, 2013;3(11):28. DOI: 10.5430/jnep.

9. Moore ZE and Cowman S. Risk assessment tools for the prevention of pressure ulcers. Cochrane Database Syst Rev. 2008;(3):CD006471.

10. Chou R, Dana T and Bougatsos C. et al editors. Pressure ulcer risk assessment and prevention: comparative effectiveness. An International Journal;18(4).

11. Baumgarten M, Margolis DJ, Localio AR, Kagan SH, et al editors. Pressure ulcers among elderly patients early in the hospital stay. J Gerontol A Biol Sci Med Sci. 2006;61(7):749-754.

12. Bergstrom N, Horn SD, Smout RJ, Bender SA et al editors. The National Pressure Ulcer Long Term Care Study: outcomes of pressure ulcer treatments in long term care. J Am Geriatr Soc. 2005;53(10):1721-1729.

13. Capon A, Pavoni N, Mastromattei A, Di Lallo D. Pressure ulcer risk in long term units: prevalence and associated factors. J Adv Nurs. 2007;58(3):263-272.

14. Pancorbo Hidalgo PL, Garcia Fernandez FP, Lopez Medina IM, Alvarez Nieto C. Risk assessment scales for pressure ulcer prevention: a systematic review. J Adv Nurs. 2006;54(1):94-110.

15. Sewchuk D, Padula C, Osborne E. Prevention and early detection of pressure ulcers in patients undergoing cardiac surgery. AORN J. 2006;84(1):75-96. 